\title{
The Effects of Previous Grazing on the Subsequent Nutrient Supply of Ungulates Grazing Late-summer Mixed-Conifer Rangelands
}

\author{
Daalkhaijav Damiran ${ }^{1}$, Timothy DelCurto ${ }^{2}$, Scott L. Findholt ${ }^{3}$, Bruce K. Johnson ${ }^{3} \&$ Martin Vavra $^{4}$ \\ ${ }^{1}$ Department of Animal and Poultry Science, University of Saskatchewan, Saskatoon, SK, S7N 5A8, Canada \\ ${ }^{2}$ Department of Animal \& Range Sciences, Montana State University, Bozeman, MT 59717, USA \\ ${ }^{3}$ Oregon Department of Fish and Wildlife, La Grande, Oregon 97850, USA \\ ${ }^{4}$ Pacific Northwest Research Station, USDA Forest Service, Forestry and Range Sciences Laboratory, La Grande, \\ OR 97850, USA \\ Correspondence: Daalkhaijav Damiran, Department of Animal and Poultry Science, University of Saskatchewan, \\ 51 Campus Drive, Saskatoon, SK S7N 5A8, Canada. Tel: 306-966-4173. E-mail: daal.damiran@ usask.ca
}

Received: July 22, 2019 Accepted: August 9, 2019 Online Published: August 28, 2019

doi:10.5539/sar.v8n4p13 URL: https://doi.org/10.5539/sar.v8n4p13

\begin{abstract}
Ecological, societal, and political discussions abound regarding intra- and inter-specific competition for nutrients among wild and domestic ungulates grazing shared forested rangelands in summer as cascading effects of prior grazing drive subsequent grazing patterns and nutrient intake. Our objective was to determine diet quality and quantity of cattle (Bos taurus), mule deer (Odocoileus hemionus hemionus; deer), and elk (Cervus elaphus nelsoni) in late-summer in response to early-summer forage utilization by cattle and elk in two consecutive years. Four 2.25 ha enclosures were constructed in previously logged mixed-conifer rangelands dominated by grand fir (Abies grandis [Douglas] Forbes.), and within each enclosure, a 0.75 ha paddock was either: 1) ungrazed, 2) grazed by cattle, or 3) grazed by elk in mid-June and mid-July at a moderate utilization level (31.9 $\pm 2.7 \%)$. After grazing treatments, each paddock was subdivided into three 0.25 ha sub-paddocks and sixteen 20 -minute foraging bouts were conducted in each sub-paddock using elk, deer, and cattle $(n=4)$. Within an animal species $\mathrm{CP}$ in diets did not differ $(p>0.05)$ between ungrazed paddocks and grazed paddocks; however, diet $\mathrm{CP}$ and IVDMD of each species was higher $(p<0.05)$ on cattle grazed paddocks compared to elk grazed. Regardless of treatment, cattle diets contained lower CP, IVDMD $(p<0.05)$ than did deer or elk diets, and relative to elk, deer consistently selected diets which contained higher CP $(p<0.05)$. In response to grazing, intake rate of DM, CP, ME did not change $(p>0.05)$ for any of the ungulates. The study revealed that early-summer grazing by cattle or elk at a moderate utilization level has minimal effect on the subsequent nutrient intake rate of cattle, deer, and elk foraging in mixed-conifer forests.
\end{abstract}

Keywords: grazing behaviour, forage selection, Bos taurus, Cervus elaphus, resource partitioning, Odocoileus hemionus hemionus

\section{Introduction}

Cattle are commonly grazed in forested ecosystems, and those same forested ecosystems provide substantial and important habitat for large ungulates such as elk (Cervus elaphus) and mule deer (Odocoileus hemionus hemionus) (Wisdom \& Thomas, 1996). Limited information is available, however, regarding the proper timing and level of use of forested ranges by cattle to minimize potential competition between cattle and wild ungulates, as well as the interaction and consequences of cattle grazing on remaining forage resources. Stewart, Bowyer, Kie, Cimon, and Johnson (2002), Coe et al. (2001), Coe, Johnson, Stewart, and Kie (2004) concluded that competition for forage could occur between elk and cattle in late-summer and species interactions may be stronger between elk and cattle compared to cattle and deer. Furthermore, the response of elk and deer to cattle grazing may vary seasonally depending on forage availability and quality (Peek \& Krausman, 1996; Wisdom \& Thomas, 1996). In fall, winter, and spring, elk preferred forage that cattle had lightly or moderately utilized the preceding summer (Crane, Mosley, Brewer, Torstenson, \& Tess, 2001). Also, forage quality on elk winter ranges in the interior Northwest can be improved by cattle grazing in spring (Anderson \& Scherzinger, 1975; Clark, Krueger, Bryant, \& Thomas, 2000). Others speculated (Svejcar \& Vavra, 1985; Parker, Gillingham, Hanley, \& 
Robbins, 1999; Cook et al., 2004) that forage quality and quantity may be greatest during the growing season, but it may nevertheless be insufficient to consistently satisfy high nutritional requirements of livestock and ungulates during late-summer and autumn. However, controlled replicated experiments have not been conducted or reported on effects of early-summer elk and/or cattle grazing on subsequent late-summer nutritional condition of cattle, deer, and elk. The objectives of this study were to investigate late-summer diet quality, nutrient intake rate and nutritional condition of cattle, deer, and elk in response to prior grazing by elk or cattle on mixed-conifer rangelands.

\section{Materials and Methods}

\subsection{Study Area}

The study was conducted on the Starkey (Starkey) Experimental Forest and Range (lat $45^{\circ} 15^{\prime} \mathrm{N}$, long $118^{\circ} 25^{\prime} \mathrm{W}$ ), located in the Wallowa-Whitman National Forest in the Blue Mountains of northeast Oregon. Elevation of Starkey ranged between $1120 \mathrm{~m}$ to $1500 \mathrm{~m}$ and total annual precipitation for the study years was $614 \mathrm{~mm}$ in year 1 and $449 \mathrm{~mm}$ in year 2, which was $12.2 \%$ above and $17.9 \%$ below average, respectively (National Atmospheric Deposition Program [NADP], 2012; Figure 1). The growing season lasts about 120 days, but no months are considered frost-free (Skovlin, Harris, Strickler, \& Garrison, 1976).

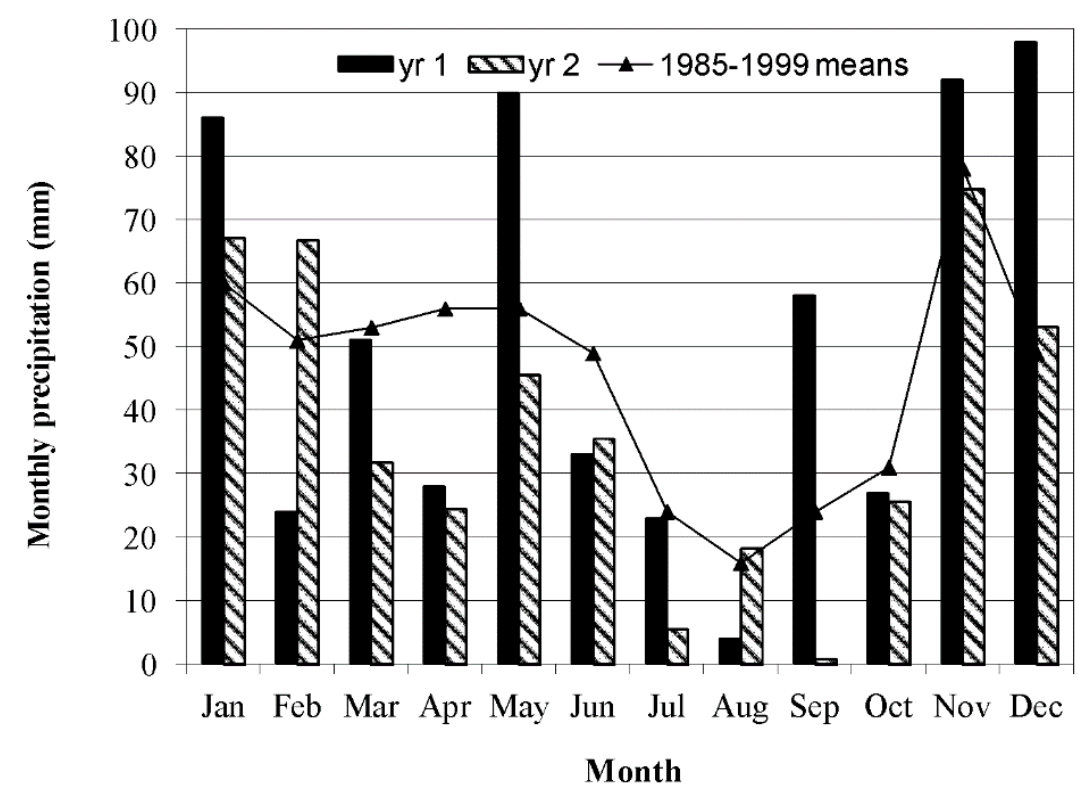

Figure 1. Monthly precipitation for yr 1 and yr 2, and long term (1985-1999) mean monthly precipitation for Starkey Experimental Forest and Range (NADP, 2012), northeast Oregon, USA

Vegetation of the study area is a mixed-conifer forest of grand fir (Abies grandis [Douglas] Forbes.), ponderosa pine (Pinus ponderosa Dougl.), and Douglas-fir (Pseudotsuga menziesii [Mirbel] Franco.), with a shrub layer of mallow ninebark (Physocarpus malvaceus [Greene] Kuntze), big huckleberry (Vaccinium membranaceum Hook.), grouse huckleberry (V. scoparium Leib.), shinyleaf spirea (Spiraea betulifolia Pall. var. lucida (Douglas ex Greene) C.L. Hitchc.) and bearberry (Arctostaphylos uva-ursi L.). Pinegrass (Calamagrostis rubescens Buckl.), California brome (Bromus carinatus Hook. \& Arn.), Kentucky bluegrass (Poa pratensis L.), western fescue (Festuca occidentalis Hook.), and elk sedge (Carex geyeri Boott) were the dominant grass species. Forbs present included western yarrow (Achillea millefolium lanulosa L.), strawberries (Fragaria vesca L. and F. virginiana Duchesne.), hawkweed (Hieracium spp.), and lupine (Lupinus spp.).

\subsection{Study Site and Grazing Treatment}

Four separate enclosures were placed in previously logged (15-20 years post harvest) mixed-conifer rangelands. We chose the grand fir vegetation type because of its dominance on summer and fall ranges in the Blue Mountains and the interior western United States. Moreover, grand fir forests support high levels of forage production, particularly after logging or burning. In addition, research indicated that mule deer, elk, and cattle concentrated much of their foraging activity after mid-summer on early successional stages of logged grand fir vegetation types (Coe et al., 2001; Findholt, Johnson, Damiran, DelCurto, \& Kie, 2004). Each enclosure was 
divided into three 0.75 -ha paddocks. Paddocks were randomly assigned as either ungrazed or grazed by cattle or grazed by elk. In paddocks to be grazed, sixty plots (caged) were protected with wire cages $(1 \times 1 \mathrm{~m})$ before grazing. Grazed paddocks were foraged by either cattle or elk in mid-June and mid-July at moderate utilization level (12 ha/animal unit) which is the typical grazing practice of forested rangeland in regional forest grazing allotments (DelCurto, Porath, Parsons, \& Morrison, 2005). Immediately after the grazing treatment and prior to foraging bout trials, sixty paired plots $\left(0.25-\mathrm{m}^{2}\right)$ per paddock were clipped to ground level. All herbage (standing crop) was separated by botanical species, oven dried at $50^{\circ} \mathrm{C}$, and weighed to quantify standing crop. Total standing crop of each plot was determined by summing the aboveground biomass of all species removed from each plot and expressed in $\mathrm{kg} / \mathrm{ha}$. The difference between the caged and grazed plots represented total forage utilization (Cook \& Stubbendieck, 1986). In ungrazed paddocks, forage biomass averaged $332 \pm 43 \mathrm{~kg} / \mathrm{ha}, 335 \pm$ $28 \mathrm{~kg} / \mathrm{ha}$, and $550 \pm 83 \mathrm{~kg} / \mathrm{ha}(n=8)$ for grasses, forbs, and shrubs, respectively. Utilization level of grasses, forbs, and shrubs was $38.8 \%, 27.3 \%$, and $30.4 \%$ in cattle grazed paddocks and $27.0 \%, 22.2 \%$, and $28.5 \%$ for elk grazed paddocks, respectively $(n=8, \mathrm{SEM}=6.2)$. Overall seasonal utilization was $32.6 \%$ and $31.2 \%$ for cattle and elk grazed paddocks, respectively which indicated that our pre-grazing treatment was at the targeted level.

\subsection{Foraging Bout Trials}

After grazing treatment was implemented, each paddock within an enclosure was subdivided into three 0.25 ha sub-paddocks using take down fences. Sub-paddocks were randomly assigned to cattle, deer and elk foraging bouts trials. Diet composition and intake of animals were measured using bite-count technique as described by Wickstrom, Robbins, Hanley, Spalinger, and Parish, (1984) and Damiran, DelCurto, Findholt, Johnson, and Vavra (2012). Sixteen (4 animals $\times 4$ foraging bouts/animal) 20 -minute foraging bouts were conducted in each sub-paddock using either 29-30 month old crossbred steers (body weight $(\mathrm{BW})=454 \pm 13 \mathrm{~kg}$ ), 36-48 month old tame female deer $(\mathrm{BW}=54 \pm 5 \mathrm{~kg})$, or tame female elk ( $\mathrm{BW}=227 \pm 9 \mathrm{~kg}$ ) in mid to late August which yielded a total of 1,152 foraging bouts. The elk and steers were the same animals used during the grazing treatments. Two foraging bouts were conducted in the morning (0800-1200 hrs) and two in the afternoon (1300-1600 hrs) for each animal in each sub-paddock. In each enclosure (block), the foraging bouts trials took 3 days to complete, and 4 weeks were required to commence a whole foraging bouts trial each year. In order to control potential bias in forage quality as plants matured over time, foraging bouts took place simultaneously with all three animal species per each enclosure. Feed was not offered to animals in morning and between foraging bouts to ensure similar appetites each day. During each foraging bout, animals were allowed to roam free in one of the sub-paddocks for 20 minutes while investigators followed the animals and counted bites by forage species and recorded the counts on a portable voice recorder (Damiran et al., 2012). Bites were counted while the investigator was close (1-2 m) to the animal, thereby assuring accurate identification of the consumed forage. After completion of foraging bouts each day, animals were fed alfalfa hay at $1.5 \%$ of body weight and held overnight in corrals for the next days' foraging bouts. Plant nomenclature followed USDA Natural Resources Conservation Service (USDA NRCS, 2012).

\subsection{Forage Sampling}

Forages selected by animals during the trial were collected simultaneously by hand clipping (Cook \& Stubbendieck, 1986) and plucking (Wallis De Vries, 1995; Damiran et al., 2012). Shrubs were hand-plucked by plucking samples between the thumb and a backward-bent forefinger. Up to 200 simulated bites of each forage species per sub-paddock were collected. Samples were dried in a forced-air oven at $50^{\circ} \mathrm{C}$ and weighed. We estimated bite size (BS) from each forage species separately for each ungulate by dividing total weight of simulated bites by total bite number.

Animal bite-count derived diet dry matter intake (DMI) calculated as:

$$
D M I(g / m i n)=\sum N_{i} B S_{i}
$$

Percent (Comp, \%) contribution of each forage species (jth) in each animal diet was calculated as:

$$
\operatorname{Comp}_{j}(\%)=\left(N_{j} B S_{j} / \sum N_{i} B S_{i}\right) / 100
$$

Animal cumulative nutrient quantity (NI) from consumed diet [either crude protein (CP), acid detergent fiber (ADF), neutral detergent fiber (NDF), or dry matter digestibility (IVDMD)] was calculated as (Damiran et al., 2012):

$$
N I,(g / m i n)=\sum N_{i} B S_{i}\left(F Q_{i} / 100\right)
$$

Animal bite-count derived diet quality (DQ) (CP, ADF, NDF, or IVDMD) was calculated as: 


$$
D Q(\%)=\left[\sum N_{i} B S_{i}\left(F Q_{i} / 100\right) / \sum N_{i} B S_{i}\right] 100
$$

Where, $\mathrm{N}_{j}$ is the number of bites of ${ }_{j}$ th forage species counted during foraging trial $(\mathrm{n} / \mathrm{min}), \mathrm{BS}_{j}$ is simulated bite size of $\mathrm{jth}$ forage species $(\mathrm{n} / \mathrm{min}), \mathrm{N}_{i}$ is the number of bites of each forage species counted during foraging trial $(\mathrm{n} / \mathrm{min}), \mathrm{BS}_{i}=$ simulated bite size of each forage species $i(\mathrm{~g}, \mathrm{DM})$, and $\mathrm{FQ}_{i}=$ nutrient composition (analyzed) of each forage species $i(\%, \mathrm{DM})$.

\subsection{Diet Quality Assay}

A total of 462 forage samples were analyzed for chemical composition after grinding them through $1 \mathrm{~mm}$ screen (Wiley Mill, Model 4, Arthur H. Thomas Co., Philadelphia, PA, USA) according to AOAC (1990) for DM (AOAC method \# 930.15), CP (AOAC method \# 984.13) by the Kjeldahl method using a Kjeltec Auto System (Kjeltec Auto System, Büchi, Flawil, Switzerland), ADF and NDF with heat stable $\alpha$-amylase according to the procedures of Van Soest et al. (1991) using ANKOM Fiber Analyzer (ANKOM Technology Corporation., Fairport, NY). Dry matter digestibility was determined using a Daisy ${ }^{\text {II }}$ incubator (ANKOM Technology Corporation, Fairport, NY) as described by Damiran, DelCurto, Bohnert, and Findholt (2008). In order to prepare a buffer-inoculum mixture as described by Marten and Barnes (1980), ruminal inoculum was obtained from two rumen-cannulated steers consuming a moderate quality ( $86 \mathrm{~g} / \mathrm{kg} \mathrm{CP}, 690 \mathrm{~g} / \mathrm{kg} \mathrm{NDF}$; DM basis) meadow hay diet. Steers were cared for in accordance with the guidelines established by the Institutional Animal Care and Use Committee at Oregon State University. Analyses were conducted with two replicates and acceptable coefficients of variation of means were $<0.5,<2.0,<3.0,<3.0$, and $<4.9 \%$ for DM, CP, ADF, NDF, and IVDMD, respectively. Chemical content and digestibility were presented on a DM basis. In vitro DMD was converted to digestible energy (DE) with the formula of Rittenhouse, Streeter, and Clanton (1971): DE (kJ/kg) = $\{[0.038 \times \operatorname{IVDMD}(\%)+0.18] \times 4.18 \times 1000\}$, and DE was converted to metabolizable energy $(\mathrm{ME})$ using the relationship provided by NRC (1996): ME $(\mathrm{kJ} / \mathrm{kg})=\mathrm{DE} \times 0.82$. Based on the bite size, bite number, and chemical composition of each forage species per each animal species, the cumulative chemical composition by growth form of plants were calculated.

\subsection{Statistical Analysis}

Response variables were analyzed as split-plots with 4 blocks per treatment combination with grazing treatment (grazing; three levels) as the main effects, and animal species (animal; three levels) and grazing $\times$ animal interactions as sub-plot effects using the mixed model procedure of SAS (SAS, 2002). Year was considered as a random effect. When a significant $F$-value was found ( $p<0.05$ ), then a Tukey-Kramer post-test (SAS, 2002) was performed. LSMeans were computed and statistically separated with the PDIFF option of SAS. Results were considered significant at $p<0.05$. Analysis showed that for the animal diet botanical composition, chemical composition, diet quality, the effect of grazing and animal species was significant; however, grazing $\times$ animal species were not significant $(p>0.05)$, and hence, grazing $\times$ animal species interaction were removed from the model and data were re-analyzed to assess grazing and animal species main effect of response variable. Likewise, for the animal nutrient intake, animal species was significant; however, grazing and grazing $\times$ animal species were not significant $(p>0.05)$, and hence, grazing and grazing $\times$ animal species interaction were removed from the model and data were re-analyzed to assess only animal species main effect of nutrient intake.

\section{Results}

\subsection{Diet Botanical Composition}

Cattle, deer, and elk utilized 68+, 84+, and 77+ forage plant species, respectively, and throughout foraging bouts 109 forage plant species were recorded in the diets (Damiran, 2006). Although, as presented in Table 1, only 36 species contributed $>5 \%$ of the diet of at least 1 animal species in at least one experimental unit (sub-paddock).

Strawberries and bearberry were greater $(p<0.05)$ in the diets in previously cattle grazed and elk grazed paddocks, respectively. In response to prior grazing (of both cattle and elk), northwestern sedge (Carex concinnoides Mack.) increased $(p<0.05)$ in the diets (Table 1). 
Table 1. Ungulates diet botanical composition (\%) on mixed-conifer rangelands at the Starkey Experimental Forest and Range, northeast Oregon, USA during late-summer grazing on ungrazed, prior cattle grazed, and elk grazed paddocks

\begin{tabular}{|c|c|c|c|c|c|}
\hline \multirow[b]{2}{*}{ Forage Species $^{1}$} & \multicolumn{3}{|c|}{ Grazing } & \multirow[b]{2}{*}{ SEM } & \multirow[b]{2}{*}{$p$-value ${ }^{2}$} \\
\hline & Ungrazed & Cattle grazed & Elk grazed & & \\
\hline Grass and Sedge & & & & & \\
\hline Bromus carinatus & 6.01 & 3.66 & 5.71 & 1.430 & 0.217 \\
\hline Carex concinnoides & $0.33^{\mathrm{b}}$ & $0.90^{\mathrm{a}}$ & $1.04^{\mathrm{a}}$ & 0.222 & 0.026 \\
\hline Carex geyeri & 11.50 & 7.44 & 6.66 & 3.301 & 0.529 \\
\hline Calamagrostis rubescens & 10.97 & 9.72 & 20.53 & 5.585 & 0.178 \\
\hline Dactylis glomerata & 8.33 & 2.70 & 3.67 & 3.236 & 0.188 \\
\hline Festuca occidentalis & 2.24 & 2.46 & 8.88 & 4.444 & 0.442 \\
\hline Phleum pratense & 1.77 & 0.26 & 0.16 & 0.599 & 0.128 \\
\hline $\begin{array}{c}\text { Poa pratensis } \\
\text { Forb }\end{array}$ & 4.79 & 2.12 & 2.42 & 1.898 & 0.336 \\
\hline Achillea millefolium lanulosa & 1.18 & 1.12 & 1.47 & 0.512 & 0.742 \\
\hline Antennaria luzuloides & 0.13 & 0.48 & 0.37 & 0.128 & 0.111 \\
\hline Arnica cordifolia & 0.28 & 0.33 & 0.17 & 0.128 & 0.441 \\
\hline Cirsium vulgare & 0.94 & 1.01 & 1.12 & 0.345 & 0.887 \\
\hline Epilobium angustifolium & 0.78 & 2.15 & 0.20 & 1.120 & 0.489 \\
\hline Epilobium minutum & 1.66 & 1.39 & 0.99 & 0.730 & 0.696 \\
\hline Epilobium paniculatum & 2.39 & 3.39 & 1.91 & 1.893 & 0.579 \\
\hline Fragaria sp. & $8.33^{\mathrm{b}}$ & $12.40^{\mathrm{a}}$ & $7.08^{\mathrm{b}}$ & 1.697 & 0.011 \\
\hline Hieracium albertinum & 1.63 & 1.43 & 0.67 & 0.591 & 0.256 \\
\hline Lupinus laxiflorus & 3.05 & 5.17 & 5.13 & 1.769 & 0.492 \\
\hline Lupinus sericeus & 0.33 & 0.99 & 1.33 & 0.552 & 0.385 \\
\hline Potentilla gracilis & 0.96 & 1.04 & 0.02 & 0.505 & 0.177 \\
\hline Thermopsis montana & 0.24 & 0.11 & 0.16 & 0.185 & 0.819 \\
\hline Tragopogon dubius & 0.39 & 0.09 & 0.08 & 0.194 & 0.372 \\
\hline $\begin{array}{l}\text { Trifolium repens } \\
\text { Shrub and Tree }\end{array}$ & 1.19 & 0.19 & 0.32 & 0.430 & 0.205 \\
\hline Amelanchier alnifolia & 0.49 & 0.63 & 0.26 & 0.296 & 0.534 \\
\hline Arctostaphylos uva-ursi & $5.83^{\mathrm{b}}$ & $7.77^{\mathrm{b}}$ & $13.67^{\mathrm{a}}$ & 1.911 & 0.002 \\
\hline Berberis repens & 1.02 & 1.29 & 0.18 & 0.428 & 0.071 \\
\hline Ceanothus velutinus & 0.15 & 0.32 & 1.36 & 0.786 & 0.372 \\
\hline Linnaea borealis & 0.44 & 2.21 & 3.04 & 1.882 & 0.306 \\
\hline Pinus ponderosa & 0.59 & 0.70 & 0.61 & 0.378 & 0.958 \\
\hline Rosa gymnocarpa & 3.66 & 4.28 & 1.40 & 1.208 & 0.119 \\
\hline Salix scouleriana & 1.19 & 1.75 & 0.37 & 0.874 & 0.428 \\
\hline Spiraea betulifolia & 2.82 & 3.05 & 2.70 & 1.278 & 0.929 \\
\hline Symphoricarpos albus & 2.07 & 3.17 & 1.51 & 1.220 & 0.082 \\
\hline Vaccinium membranaceum & 1.78 & 4.09 & 0.88 & 1.351 & 0.104 \\
\hline $\begin{array}{l}\text { Vaccinium scoparium } \\
\text { Lichen }\end{array}$ & 0.16 & 0.73 & 0.65 & 0.469 & 0.413 \\
\hline Bryoria fremontii & 7.55 & 8.14 & 2.19 & 2.291 & 0.077 \\
\hline Other forages & $2.84^{\mathrm{a}}$ & $1.35^{\mathrm{b}}$ & $1.07^{\mathrm{b}}$ & 0.375 & 0.001 \\
\hline
\end{tabular}

Note. ${ }^{1}$ Forage species that made up $\geq 5 \%$ of the diet of at least 1 animal species in at least one sub-paddock are included.

${ }^{2}$ There was no grazing $\times$ animal interaction $(p>0.05)$.

${ }^{\mathrm{ab}}$ Row values with different superscripts differ $(p<0.05, n=12)$. 
Table 2. Cattle, deer, and elk diet botanical composition (\%) on mixed-conifer rangelands at the Starkey Experimental Forest and Range, northeast Oregon, USA during late-summer grazing

\begin{tabular}{|c|c|c|c|c|c|}
\hline \multirow[b]{2}{*}{ Forage Species $^{1}$} & \multicolumn{3}{|c|}{ Animal Species } & \multirow[b]{2}{*}{ SEM } & \multirow[b]{2}{*}{$p$-value ${ }^{2}$} \\
\hline & Cattle & Deer & Elk & & \\
\hline \multicolumn{6}{|l|}{ Grass and Sedge } \\
\hline Bromus carinatus & $10.89^{\mathrm{a}}$ & $1.02^{\mathrm{b}}$ & $3.47^{\mathrm{b}}$ & 1.430 & $<0.001$ \\
\hline Carex concinnoides & $0.53^{\mathrm{b}}$ & $0.40^{\mathrm{b}}$ & $1.34^{\mathrm{a}}$ & 0.222 & 0.002 \\
\hline Carex geyeri & $16.96^{\mathrm{a}}$ & $0.58^{\mathrm{c}}$ & $8.07^{\mathrm{b}}$ & 2.507 & $<0.001$ \\
\hline Calamagrostis rubescens & $26.17^{\mathrm{a}}$ & $0.90^{\mathrm{c}}$ & $14.16^{\mathrm{b}}$ & 5.075 & $<0.001$ \\
\hline Dactylis glomerata & $5.16^{\mathrm{ab}}$ & $2.03^{\mathrm{b}}$ & $7.50^{\mathrm{a}}$ & 2.986 & 0.016 \\
\hline Festuca occidentalis & $8.97^{\mathrm{a}}$ & $0.01^{\mathrm{b}}$ & $4.59^{\mathrm{ab}}$ & 3.386 & $<0.001$ \\
\hline Phleum pratense & $1.07^{\mathrm{a}}$ & $0.08^{\mathrm{b}}$ & $1.04^{\mathrm{a}}$ & 0.473 & 0.017 \\
\hline Poa pratensis & $8.23^{\mathrm{a}}$ & $0.79^{\mathrm{b}}$ & $0.30^{\mathrm{b}}$ & 1.831 & $<0.001$ \\
\hline \multicolumn{6}{|l|}{ Forb } \\
\hline Achillea millefolium lanulosa & $0.39^{\mathrm{b}}$ & $3.23^{\mathrm{a}}$ & $0.15^{\mathrm{b}}$ & 0.512 & $<0.001$ \\
\hline Antennaria luzuloides & $0.20^{\mathrm{b}}$ & $0.12^{\mathrm{b}}$ & $0.66^{\mathrm{a}}$ & 0.128 & 0.004 \\
\hline Arnica cordifolia & 0.19 & 0.34 & 0.26 & 0.128 & 0.484 \\
\hline Cirsium vulgare & $1.04^{\mathrm{b}}$ & $0.03^{\mathrm{c}}$ & $2.00^{\mathrm{a}}$ & 0.339 & 0.001 \\
\hline Epilobium angustifolium & 0.02 & 1.65 & 1.45 & 0.849 & 0.182 \\
\hline Epilobium minutum & $0.37^{\mathrm{b}}$ & $2.71^{\mathrm{a}}$ & $0.97^{\mathrm{ab}}$ & 0.730 & 0.011 \\
\hline Epilobium paniculatum & $0.00^{\mathrm{b}}$ & $6.77^{\mathrm{a}}$ & $0.93^{\mathrm{b}}$ & 1.893 & $<0.001$ \\
\hline Fragaria sp. & $2.75^{\mathrm{c}}$ & $14.99^{\mathrm{a}}$ & $10.06^{\mathrm{b}}$ & 1.697 & $<0.001$ \\
\hline Hieracium albertin & 0.91 & 1.64 & 1.18 & 0.543 & 0.132 \\
\hline Lupinus laxiflorus & 4.81 & 3.87 & 4.67 & 1.694 & 0.836 \\
\hline Lupinus sericeus & $0.00^{\mathrm{b}}$ & $2.61^{\mathrm{a}}$ & $0.04^{\mathrm{b}}$ & 0.552 & $<0.001$ \\
\hline Potentilla gracilis & $0.00^{\mathrm{b}}$ & $1.24^{\mathrm{a}}$ & $0.79^{\mathrm{a}}$ & 0.442 & $<0.001$ \\
\hline Thermopsis montana & 0.02 & 0.48 & 0.00 & 0.185 & 0.034 \\
\hline Tragopogon dubius & $0.02^{\mathrm{b}}$ & $0.41^{\mathrm{a}}$ & $0.13^{\mathrm{ab}}$ & 0.157 & 0.005 \\
\hline Trifolium repens & 0.44 & 0.20 & 1.06 & 0.428 & 0.249 \\
\hline \multicolumn{6}{|l|}{ Shrub and Tree } \\
\hline Amelanchier alnifolia & $0.01^{\mathrm{b}}$ & $1.27^{\mathrm{a}}$ & $0.09^{\mathrm{b}}$ & 0.279 & $<0.001$ \\
\hline Arctostaphylos uva-ursi & $0.62^{\mathrm{c}}$ & $19.87^{\mathrm{a}}$ & $6.77^{\mathrm{b}}$ & 1.911 & $<0.001$ \\
\hline Berberis repens & $0.72^{\mathrm{b}}$ & $0.09^{\mathrm{b}}$ & $1.68^{\mathrm{a}}$ & 0.405 & $<0.001$ \\
\hline Ceanothus velutinus & 0.16 & 1.50 & 0.16 & 0.786 & 0.253 \\
\hline Linnaea borealis & $1.94^{\mathrm{ab}}$ & $0.14^{\mathrm{b}}$ & $3.62^{\mathrm{a}}$ & 1.823 & 0.039 \\
\hline Pinus ponderosa & $0.00^{\mathrm{b}}$ & $1.90^{\mathrm{a}}$ & $0.00^{\mathrm{b}}$ & 0.378 & $<0.001$ \\
\hline Rosa gymnocarpa & $0.22^{\mathrm{b}}$ & $7.16^{\mathrm{a}}$ & $1.96^{\mathrm{b}}$ & 1.193 & $<0.001$ \\
\hline Salix scouleriana & $0.13^{\mathrm{b}}$ & $2.91^{\mathrm{a}}$ & $0.26^{\mathrm{b}}$ & 0.844 & 0.004 \\
\hline Spiraea betulifolia & $2.17^{\mathrm{b}}$ & $4.77^{\mathrm{a}}$ & $1.63^{\mathrm{b}}$ & 1.278 & 0.003 \\
\hline Symphoricarpos albus & 1.78 & 2.24 & 2.73 & 1.213 & 0.245 \\
\hline Vaccinium membranaceum & 1.10 & 2.80 & 2.85 & 1.222 & 0.048 \\
\hline $\begin{array}{l}\text { Vaccinium scoparium } \\
\text { Lichen }\end{array}$ & 0.35 & 0.58 & 0.61 & 0.436 & 0.653 \\
\hline Bryoria fremontii & $0.31^{\mathrm{c}}$ & $5.62^{\mathrm{b}}$ & $11.95^{\mathrm{a}}$ & 2.202 & $<0.001$ \\
\hline Other forages & $1.35^{\mathrm{b}}$ & $3.06^{\mathrm{a}}$ & $0.85^{\mathrm{b}}$ & 0.375 & $<0.001$ \\
\hline
\end{tabular}

Note. ${ }^{1}$ Forage species that made up $\geq 5 \%$ of the diet of at least 1 animal species in at least one sub-paddock are included.

${ }^{2}$ There was no grazing $\times$ animal interaction $(p>0.05)$.

${ }^{\mathrm{abc}}$ Row values with different superscripts differ $(p<0.05, n=12)$.

Cattle selected greater $(p<0.05)$ California brome, elk sedge, pinegrass, and Kentucky bluegrass, but less $(p<$ 0.05 ) bearberry and Fremont's horsehair lichen (Bryoria fremontii (Tuck.) Brodo \& D. Hawksw.) compared to deer or elk, while cattle were similar $(p>0.05)$ to both elk and deer on orchardgrass (Dactylis glomerata L.) selection in the diet (Table 2). Compared to deer, cattle selected greater $(p<0.05)$ western fescue, timothy 
(Phleum pratense L.), and bull thistle (Cirsium vulgare (Savi) Ten.), but selected less $(p<0.05$ ) western yarrow, chaparral willowherb (Epilobium minutum Lindl. ex Lehm.), silky lupine (Lupinus sericeus Pursh), yellow salsify (Tragopogon dubius Scop.), Saskatoon serviceberry (Amelanchier alnifolia (Nutt.) Nutt. ex M. Roem.), ponderosa pine, baldhip rose (Rosa gymnocarpa Nutt.), Scouler's willow (Salix scouleriana Barratt ex Hook.), and shinyleaf spirea. Cattle and deer were, however, similar $(p>0.05)$ in selecting northwestern sedge, rush pussytoes (Antennaria luzuloides Torr. \& A. Gray), creeping barberry (Berberis repens Lindl.), and twinflower (Linnaea borealis L.) (Table 2). Compared to elk, cattle chosen less $(p<0.05)$ rush pussytoes, bull thistle, beauty cinquefoil (Potentilla gracilis Douglas ex Hook.), and creeping barberry, but selected similar amount of ( $p>$ 0.05) timothy, western fescue, western yarrow, chaparral willowherb, silky lupine, yellow salsify, Saskatoon serviceberry, twinflower, baldhip rose, and shinyleaf spirea (Table 2). Compared to elk, deer selected greater amount of $(p<0.05)$ western yarrow, fireweed (Epilobium angustifolium L.), strawberry spp., silky lupine, Saskatoon serviceberry, bearberry, ponderosa pine, baldhip rose, Scouler's willow, and shinyleaf spirea (Table 2).

\subsection{Quality of Forage}

Chemical composition of forages selected by animals were pooled by growth form and presented in Table 3 by grazing treatment and in Table 4 by animal species. Grazing was not interacted (Table 3; $p>0.05$ ); but animal species (Table $4 ; p<0.05)$ interacted with forage quality. Cattle selected grasses with lower $(p<0.05) \mathrm{CP}$ and IVDMD, and greater $(p<0.05)$ fiber (ADF and NDF) than did deer or elk. However, cattle-selected sedges were similar $(p>0.05)$ in nutritional quality with that of elk, but lower $(p<0.05)$ in CP and IVDMD and greater $(p<$ $0.05)$ in fiber compared to deer diets. Cattle selected forbs with lower $(p<0.05) \mathrm{CP}$, but greater $(p<0.05)$ fiber and IVDMD than deer; however they were similar $(p>0.05)$ in CP and IVDMD and greater $(p<0.05)$ in fiber than forbs selected by elk. Cattle selected shrub diets greater $(p<0.05)$ in NDF and IVDMD, but similar $(p>$ $0.05)$ in CP to deer diets. All three animal species had shrub diets similar $(p>0.05)$ in ADF. Moreover, cattleand elk-selected shrubs had similar $(p>0.05)$ NDF and IVDMD (Table 4). Deer-selected grass, sedge, and forbs were greater $(p<0.05)$ in CP; whereas grass, sedge, and lichen were greater $(p<0.05)$ in IVDMD than elk selected. However, forbs and shrubs in deer and elk diets did not differ $(p>0.05)$ in CP and IVDMD (Table 4).

\subsection{Diet Quality and Quantity}

Grazing and animal effects were detected $(p<0.05)$ for diet quality (Table 5). Diet CP level of all three animal species was higher $(p<0.05)$ on the prior cattle grazed paddocks compared to prior elk grazed paddocks. However, CP levels of diets were not different $(p>0.05)$ on prior cattle or elk grazed paddocks compared to ungrazed paddocks.

Acid detergent fiber of diets was the greatest $(p<0.05)$, while IVDMD and ME were the lowest $(p<0.05)$ on prior elk grazed paddocks. Response of diet ADF under prior cattle and elk grazing varied. Under prior elk grazing, NDF levels did not change $(p>0.05)$ relative to diets selected in ungrazed paddocks. In contrast, diet NDF levels were lower $(p<0.05)$ on prior cattle grazed paddocks compared to ungrazed or prior elk grazed paddocks.

Cattle diets contained lower $(p<0.05)$ CP, IVDMD, and ME, but higher ADF and NDF compared to deer or elk diets. Relative to elk, deer consistently selected forages containing higher $\mathrm{CP}(p<0.05)$, and lower $(p<0.05)$ ADF and NDF. Digestibility (IVDMD) and ME levels of deer and elk diets were similar (Table 5; $p>0.05$ ).

Deer CPI, DMI, and MEI were lower $(p<0.01 ; n=24)$ than those of cattle and elk which had similar $(p>0.05)$ nutrient intake rates (Table 6). Bite size was 661,160 , and $471 \mathrm{mg} / \mathrm{bite}(\mathrm{SEM}=59 \mathrm{mg}$ ) for cattle, deer, and elk, respectively (data not shown).

Changes in the contribution (\%) of growth form of forages were found for cattle and elk but not deer across treatments in CP and ME intake (Table 7). The largest differences were for cattle within cattle grazed treatment with decreased grass contribution $(p<0.05)$ and increased forb contribution $(p<0.05)$ compared to the ungrazed paddocks. For elk, grass contributed less to total dietary intake of $\mathrm{CP}$ and ME from $(p<0.05)$ previously cattle grazed paddocks, and lichen decreased $(p<0.05)$ for $\mathrm{CP}$ and ME intake in previously elk grazed paddocks (Table 7). 
Table 3. Cattle, deer, and elk diet chemical composition (\%, DM) on mixed-conifer rangelands at the Starkey Experimental Forest and Range, northeast Oregon, USA during late-summer grazing on ungrazed, prior cattle grazed, and elk grazed paddocks

\begin{tabular}{llllll}
\hline & & & & & \\
Item $^{1}$ & Grazing & & & \\
\cline { 2 - 4 } CP & & & & & \\
Gngrazed & Cattle grazed & Elk grazed & SEM & $p$-value \\
Grass & 7.03 & 7.09 & 7.73 & 0.368 & 0.139 \\
Sedge & 6.92 & 6.82 & 6.84 & 0.153 & 0.887 \\
Forb & 9.19 & 9.75 & 9.77 & 0.233 & 0.127 \\
Shrub $^{2}$ & 8.17 & 8.11 & 7.33 & 0.369 & 0.080 \\
Lichen $^{3}$ & 9.96 & 9.89 & 10.00 & 0.279 & 0.961 \\
ADF & & & & & \\
Grass & $40.37^{\mathrm{a}}$ & $41.81^{\mathrm{a}}$ & $42.30^{\mathrm{b}}$ & 0.870 & 0.046 \\
Sedge & 40.22 & 39.38 & 40.75 & 0.875 & 0.473 \\
Forb & 29.32 & 27.49 & 30.21 & 1.210 & 0.086 \\
Shrub & 29.60 & 30.10 & 32.81 & 1.471 & 0.075 \\
Lichen & 11.34 & 10.81 & 11.22 & 1.585 & 0.967 \\
NDF & & & & & \\
Grass & 58.79 & 59.08 & 59.13 & 1.404 & 0.934 \\
Sedge & 59.02 & 60.07 & 60.55 & 1.186 & 0.532 \\
Forb & 35.65 & 34.05 & 36.75 & 1.537 & 0.220 \\
Shrub & 34.30 & 34.23 & 36.00 & 0.921 & 0.189 \\
Lichen & 37.77 & 37.59 & 37.85 & 1.070 & 0.984 \\
IVDMD & & & & & \\
Grass & 57.11 & 55.90 & 55.92 & 1.402 & 0.562 \\
Sedge & 56.17 & 57.30 & 56.52 & 0.534 & 0.306 \\
Forb & 61.01 & 60.60 & 62.67 & 1.346 & 0.207 \\
Shrub & 59.23 & 58.58 & 55.39 & 1.240 & 0.098 \\
Lichen & 66.26 & 66.54 & 66.17 & 1.548 & 0.984 \\
\hline
\end{tabular}

Note. ${ }^{1}$ Composition of forage selected based on bite number, bite size, and nutrient composition of each forage species consumed by animals during foraging bouts.

${ }^{\mathrm{ab}}$ Row values with different superscripts differ $(p<0.05, n=12)$.

${ }^{2}$ Tree species included in deer diet.

${ }^{3}$ Fremont's horsehair lichen (Bryoria fremontii (Tuck.) Brodo \& D. Hawksw.).

${ }^{4}$ There was no grazing $\times$ animal interaction $(p>0.05)$. 
Table 4. Cattle, deer, and elk diet chemical composition (\%, DM) on mixed-conifer rangelands at the Starkey Experimental Forest and Range, northeast Oregon USA during late-summer grazing

\begin{tabular}{|c|c|c|c|c|c|}
\hline \multirow[b]{2}{*}{ Item $^{1}$} & \multicolumn{3}{|c|}{ Animal Species } & \multirow[b]{2}{*}{ SEM } & \multirow[b]{2}{*}{$p$-value ${ }^{4}$} \\
\hline & Cattle & Deer & Elk & & \\
\hline \multicolumn{6}{|l|}{$\mathrm{CP}$} \\
\hline Grass & $6.17^{\mathrm{c}}$ & $8.57^{\mathrm{a}}$ & $7.11^{\mathrm{b}}$ & 0.368 & $<0.001$ \\
\hline Sedge & $6.08^{\mathrm{b}}$ & $8.14^{\mathrm{a}}$ & $6.35^{\mathrm{b}}$ & 0.155 & $<0.001$ \\
\hline Forb & $9.42^{\mathrm{b}}$ & $10.37^{\mathrm{a}}$ & $8.91^{\mathrm{b}}$ & 0.232 & $<0.001$ \\
\hline Shrub $^{2}$ & $8.13^{\mathrm{a}}$ & $7.96^{\mathrm{ab}}$ & $7.53^{\mathrm{b}}$ & 0.340 & 0.023 \\
\hline Lichen $^{3}$ & $11.23^{\mathrm{a}}$ & $9.37^{\mathrm{b}}$ & $9.25^{\mathrm{b}}$ & 0.285 & $<0.001$ \\
\hline \multicolumn{6}{|l|}{$\mathrm{ADF}$} \\
\hline Grass & $45.17^{\mathrm{a}}$ & $37.49^{c}$ & $41.81^{b}$ & 0.870 & $<0.001$ \\
\hline Sedge & $42.04^{\mathrm{a}}$ & $37.22^{\mathrm{b}}$ & $41.08^{\mathrm{a}}$ & 0.825 & $<0.001$ \\
\hline Forb & $33.28^{\mathrm{a}}$ & $27.54^{\mathrm{b}}$ & $26.19^{\mathrm{b}}$ & 1.210 & $<0.001$ \\
\hline Shrub & $30.06^{\mathrm{a}}$ & $31.25^{\mathrm{a}}$ & $31.20^{\mathrm{a}}$ & 1.427 & 0.435 \\
\hline Lichen & $5.13^{\mathrm{b}}$ & $7.70^{\mathrm{b}}$ & $20.55^{\mathrm{a}}$ & 1.616 & $<0.001$ \\
\hline \multicolumn{6}{|l|}{ NDF } \\
\hline Grass & $62.64^{\mathrm{a}}$ & $56.35^{\mathrm{b}}$ & $58.02^{\mathrm{b}}$ & 1.404 & $<0.001$ \\
\hline Sedge & $61.83^{\mathrm{a}}$ & $55.48^{\mathrm{b}}$ & $62.33^{\mathrm{a}}$ & 1.169 & $<0.001$ \\
\hline Forb & $41.24^{\mathrm{a}}$ & $33.79^{\mathrm{b}}$ & $31.41^{\mathrm{b}}$ & 1.477 & $<0.001$ \\
\hline Shrub & $36.20^{\mathrm{a}}$ & $32.50^{\mathrm{b}}$ & $35.83^{\mathrm{a}}$ & 0.876 & $<0.001$ \\
\hline Lichen & $34.84^{\mathrm{b}}$ & $38.55^{\mathrm{ab}}$ & $39.81^{\mathrm{a}}$ & 1.091 & 0.012 \\
\hline \multicolumn{6}{|l|}{ IVDMD } \\
\hline Grass & $52.62^{\mathrm{c}}$ & $60.20^{\mathrm{a}}$ & $56.11^{\mathrm{b}}$ & 1.402 & $<0.001$ \\
\hline Sedge & $55.55^{\mathrm{b}}$ & $58.88^{\mathrm{a}}$ & $55.56^{\mathrm{b}}$ & 0.538 & $<0.001$ \\
\hline Forb & $63.21^{\mathrm{a}}$ & $59.74^{\mathrm{b}}$ & $61.33^{\mathrm{ab}}$ & 1.333 & 0.005 \\
\hline Shrub & $59.41^{\mathrm{a}}$ & $56.55^{\mathrm{b}}$ & $57.25^{\mathrm{ab}}$ & 1.082 & 0.039 \\
\hline Lichen & $70.66^{\mathrm{a}}$ & $69.26^{\mathrm{a}}$ & $59.04^{\mathrm{b}}$ & 1.579 & $<0.001$ \\
\hline
\end{tabular}

Note. ${ }^{1}$ Composition of forage selected based on bite number, bite size, and nutrient composition of each forage species consumed by animals during foraging bouts. ${ }^{\text {abc }}$ Row values with different superscripts differ $(p<0.05, n$ $=12$ ).

${ }^{2}$ Tree species included in deer diet.

${ }^{3}$ Fremont's horsehair lichen (Bryoria fremontii (Tuck.) Brodo \& D. Hawksw.).

${ }^{4}$ There was no grazing $\times$ animal interaction $(p>0.05)$.

Table 5. Comparison of changes in diet quality of cattle, deer, and elk as affected by previous cattle or elk grazing in regenerating grand fir rangeland at the Starkey Experimental Forest and Range, northeast Oregon, USA

\begin{tabular}{|c|c|c|c|c|c|c|c|c|c|}
\hline \multirow[b]{2}{*}{ Item $^{1}$} & \multicolumn{3}{|l|}{ Grazing } & \multicolumn{3}{|c|}{ Animal Species } & \multirow[b]{2}{*}{ SEM } & \multicolumn{2}{|l|}{$p$-value ${ }^{2}$} \\
\hline & Ungrazed & $\begin{array}{l}\text { Cattle } \\
\text { grazed }\end{array}$ & $\begin{array}{l}\text { Elk } \\
\text { grazed }\end{array}$ & Cattle & Deer & Elk & & Grazing & Animal \\
\hline $\mathrm{CP}(\%, \mathrm{DM})$ & $7.8^{\mathrm{ab}}$ & $8.3^{\mathrm{b}}$ & $7.6^{\mathrm{a}}$ & $6.8^{\mathrm{a}}$ & $9.0^{\mathrm{b}}$ & $7.9^{\mathrm{c}}$ & 0.32 & 0.005 & $<0.001$ \\
\hline $\operatorname{ADF}(\%, D M)$ & $33.8^{\mathrm{a}}$ & $31.8^{\mathrm{a}}$ & $37.5^{\mathrm{b}}$ & $41.5^{\mathrm{a}}$ & $29.0^{\mathrm{b}}$ & $32.6^{\mathrm{c}}$ & 0.87 & $<0.001$ & $<0.001$ \\
\hline $\operatorname{NDF}(\%, \mathrm{DM})$ & $46.3^{\mathrm{a}}$ & $42.2^{\mathrm{b}}$ & $48.1^{\mathrm{a}}$ & $57.2^{\mathrm{a}}$ & $35.0^{\mathrm{b}}$ & $44.3^{\mathrm{c}}$ & 1.21 & 0.004 & $<0.001$ \\
\hline IVDMD (\%) & $57.8^{\mathrm{a}}$ & $58.1^{\mathrm{a}}$ & $55.8^{\mathrm{b}}$ & $55.2^{\mathrm{a}}$ & $58.3^{\mathrm{b}}$ & $58.2^{\mathrm{b}}$ & 1.03 & 0.047 & 0.009 \\
\hline $\begin{array}{l}\mathrm{ME} \\
\mathrm{DM})\end{array}$ & $8.1^{\mathrm{a}}$ & $8.2^{\mathrm{a}}$ & $7.9^{\mathrm{b}}$ & $7.8^{\mathrm{a}}$ & $8.2^{\mathrm{b}}$ & $8.2^{\mathrm{b}}$ & 0.14 & 0.047 & 0.009 \\
\hline
\end{tabular}

Note. ${ }^{1}$ Crude protein $(\mathrm{CP})$, acid detergent fiber (ADF), neutral detergent fiber (NDF), in vitro dry matter digestibility (IVDMD), and metabolizable energy (ME).

${ }^{2}$ There was no grazing $\times$ animal interaction $(p>0.05)$.

${ }^{\mathrm{abc}}$ Row values within grazing treatment or animal species with different superscripts differ $(p<0.05, n=12)$. 
Table 6. Cattle, deer and elk nutrient intake rate during grazing in late-summer mixed-conifer rangelands on the Starkey Experimental Forest and Range, northeast Oregon, USA

\begin{tabular}{llllll}
\hline & \multicolumn{3}{l}{ Animal Species } & & \\
\cline { 2 - 4 } Item $^{1}$ & Cattle & Deer & Elk & SEM & $p$-value \\
\hline CPI $\left(\mathrm{g} / \mathrm{kg}^{0.75} / \mathrm{min}\right)$ & $0.012^{\mathrm{a}}$ & $0.008^{\mathrm{b}}$ & $0.011^{\mathrm{a}}$ & 0.0038 & $<0.001$ \\
DMI $\left(\mathrm{g} / \mathrm{kg}^{0.75} / \mathrm{min}\right)$ & $0.179^{\mathrm{a}}$ & $0.093^{\mathrm{b}}$ & $0.148^{\mathrm{a}}$ & 0.0131 & $<0.001$ \\
MEI $\left(\mathrm{kJ} / \mathrm{kg}^{0.75} / \mathrm{min}\right)$ & $1.380^{\mathrm{a}}$ & $0.757^{\mathrm{b}}$ & $1.212^{\mathrm{a}}$ & 0.1003 & $<0.001$ \\
\hline
\end{tabular}

Note. ${ }^{1}$ Crude protein intake (CPI), Dry matter intake (DMI), and metabolizable energy intake (MEI) rates. Digestible energy (DE) was converted to metabolizable energy (ME) using the relationship provided by NRC (1996): $\mathrm{ME}(\mathrm{kJ} / \mathrm{kg})=\mathrm{DE} \times 0.82$. Digestible energy was calculated with the formula of Rittenhouse et al. (1971): $\mathrm{DE}(\mathrm{kJ} / \mathrm{g})=[0.038 \times \operatorname{IVDMD}(\%)+0.18] \times 4.18$.

${ }^{2}$ There was no grazing and grazing $\times$ animal interaction $(p>0.05)$.

${ }^{\mathrm{ab}}$ Row values with different superscripts differ $(p<0.05, n=24)$.

\section{Discussion}

When compared to ungrazed paddocks, animal diet quality improved on prior cattle grazed paddocks but declined on elk grazed paddocks (Table 5). These contrasting findings on prior grazing effects are likely explained by different foraging strategies of these two species. During the grazing treatments, cattle removed $38.8 \%, 27.3 \%$, and $30.4 \%$ of grasses, forbs, and shrubs, respectively, while elk removed $27.0 \%, 22.2 \%$, and $28.5 \%$ of grasses, forbs, and shrubs, respectively (Damiran, 2006). During grazing, cattle primarily utilized graminoids, therefore high quality forbs and shrubs were still available over a longer period for later use in the cattle treatment at the levels of utilization we observed. In contrast, elk more uniformly selected from all forage growth forms (presumably with high quality parts), and thus paddock forage nutritive value may have decreased within a relatively short foraging period.

The challenge of free-ranging animals is to meet nutritional requirements necessary to complete life processes by finding and ingesting scarce forage with nutrient concentrations higher than their requirements and mixing it with more abundant forages with lower nutrient concentrations (Rittenhouse, 2000). In our study, graminoids contained lower CP and digestible DM, and higher cell wall carbohydrates (ADF and NDF) compared to forbs, shrubs, and lichen. These results concur with the existing literature (Holechek \& Vavra, 1983; Darambazar, DelCurto, \& Damiran, 2013) which suggested shrubs retain more CP than mature graminoids or forbs in late-summer. Also, the results of the current study were in agreement with others (Holechek \& Vavra, 1982; Findholt et al., 2004; DelCurto et al., 2005) who suggested that cattle and elk shift their diets to more forbs and shrubs to maintain their rate of intake when graminoids availability and/or palatability decline. 
Table 7. Contribution (\%) of growth form of forages on cattle, deer, and elk CP and ME intake in mixed-conifer rangelands on the Starkey Experimental Forest and Range, northeast Oregon, USA during late-summer grazing on ungrazed (UG), prior cattle grazed (CG), and elk grazed (EG) paddocks

\begin{tabular}{|c|c|c|c|c|c|c|c|c|c|c|}
\hline \multirow[b]{2}{*}{ Item $^{1}$} & \multicolumn{5}{|l|}{$\mathrm{CP}$} & \multicolumn{5}{|l|}{$\mathrm{ME}$} \\
\hline & Grass & Sedge & Forb & Shrub $^{2}$ & Lichen $^{3}$ & Grass & Sedge & Forb & Shrub & Lichen \\
\hline \multicolumn{11}{|l|}{ Cattle } \\
\hline UG & $60.51^{\mathrm{a}}$ & $24.00^{\mathrm{a}}$ & $8.75^{\mathrm{e}}$ & $6.24^{\mathrm{e}}$ & $0.50^{\mathrm{b}}$ & $63.35^{\mathrm{a}}$ & $24.02^{\mathrm{a}}$ & $6.99^{c}$ & $5.25^{\mathrm{d}}$ & $0.39^{c}$ \\
\hline CG & $35.96^{\mathrm{b}}$ & $15.60^{\mathrm{ab}}$ & $27.29^{\mathrm{bcd}}$ & $20.47^{\mathrm{de}}$ & $0.69^{b}$ & $38.42^{\mathrm{b}}$ & $17.09^{\mathrm{ab}}$ & $24.06^{\mathrm{bc}}$ & $19.85^{\mathrm{cd}}$ & $0.58^{\mathrm{c}}$ \\
\hline EG & $72.71^{\mathrm{a}}$ & $11.93^{\mathrm{ab}}$ & $10.37^{\mathrm{de}}$ & $4.70^{\mathrm{e}}$ & $0.28^{\mathrm{b}}$ & $73.94^{\mathrm{a}}$ & $12.57^{\mathrm{abc}}$ & $8.78^{c}$ & $4.48^{\mathrm{d}}$ & $0.24^{\mathrm{c}}$ \\
\hline \multicolumn{11}{|l|}{ Deer } \\
\hline UG & $5.11^{\mathrm{c}}$ & $0.37^{\mathrm{b}}$ & $48.00^{\mathrm{a}}$ & $39.88^{\mathrm{abc}}$ & $6.72^{\mathrm{ab}}$ & $5.58^{\mathrm{c}}$ & $0.33^{b c}$ & $44.29^{\mathrm{a}}$ & $42.45^{\mathrm{ab}}$ & $7.35^{\mathrm{abc}}$ \\
\hline CG & $3.06^{\mathrm{c}}$ & $0.52^{\mathrm{b}}$ & $47.86^{\mathrm{a}}$ & $41.34^{\mathrm{ab}}$ & $7.49^{\mathrm{ab}}$ & $3.49^{c}$ & $0.60^{c}$ & $43.60^{\mathrm{a}}$ & $44.13^{\mathrm{a}}$ & $8.48^{\mathrm{abc}}$ \\
\hline EG & $6.12^{\mathrm{c}}$ & $2.44^{\mathrm{b}}$ & $42.13^{\mathrm{ab}}$ & $45.78^{\mathrm{a}}$ & $3.91^{\mathrm{b}}$ & $6.31^{\mathrm{c}}$ & $2.67^{b c}$ & $38.20^{\mathrm{ab}}$ & $49.20^{\mathrm{a}}$ & $4.05^{\mathrm{bc}}$ \\
\hline \multicolumn{11}{|l|}{ Elk } \\
\hline UG & $29.46^{\mathrm{b}}$ & $9.22^{\mathrm{b}}$ & $29.90^{\mathrm{bc}}$ & $14.81^{\mathrm{de}}$ & $16.61^{\mathrm{a}}$ & $31.87^{\mathrm{b}}$ & $10.28^{\mathrm{bc}}$ & $28.03^{\mathrm{ab}}$ & $14.68^{\mathrm{cd}}$ & $15.14^{\mathrm{ab}}$ \\
\hline CG & $14.22^{\mathrm{c}}$ & $5.66^{\mathrm{ab}}$ & $35.42^{\mathrm{abc}}$ & $27.43^{\text {bcd }}$ & $17.28^{\mathrm{a}}$ & $15.56^{\mathrm{c}}$ & $6.94^{\mathrm{abc}}$ & $33.23^{\mathrm{ab}}$ & $28.12^{\mathrm{bc}}$ & $16.15^{\mathrm{a}}$ \\
\hline EG & $41.45^{\mathrm{b}}$ & $7.35^{\mathrm{ab}}$ & $23.58^{\text {cde }}$ & $23.98^{\mathrm{cd}}$ & $3.64^{\mathrm{b}}$ & $40.85^{\mathrm{b}}$ & $8.35^{\mathrm{abc}}$ & $22.06^{\mathrm{bc}}$ & $25.42^{\mathrm{c}}$ & $3.31^{\mathrm{c}}$ \\
\hline SEM & 3.204 & 4.487 & 4.749 & 4.611 & 2.96 & 3.396 & 4.421 & 4.463 & 4.675 & 2.906 \\
\hline \multicolumn{11}{|l|}{$p$-value } \\
\hline Grazing & 0.005 & 0.664 & 0.002 & 0.055 & 0.141 & 0.001 & 0.718 & 0.005 & 0.072 & 0.121 \\
\hline Animal & 0.001 & 0.001 & 0.001 & 0.001 & 0.001 & 0.001 & 0.001 & 0.001 & 0.001 & 0.001 \\
\hline Grazing $\times$ Animal & 0.001 & 0.17 & 0.205 & 0.051 & 0.062 & 0.001 & 0.208 & 0.239 & 0.056 & 0.123 \\
\hline
\end{tabular}

Note. ${ }^{1}$ Contribution of forage obtained through summing selected forage composition based on bite number, bite size, and nutrient composition of each forage species consumed by animals during foraging bouts.

${ }^{\mathrm{a}-\mathrm{c}}$ Column values with different superscripts differ $(p<0.05 ; n=8)$.

${ }^{2}$ Tree species included in deer diet.

${ }^{3}$ Fremont's horsehair lichen (Bryoria fremontii (Tuck.) Brodo \& D. Hawksw.)

${ }^{4}$ Digestible energy (DE) was converted to metabolizable energy (ME) using the relationship provided by NRC (1996): $\mathrm{ME}(\mathrm{kJ} / \mathrm{kg})=\mathrm{DE} \times 0.82$. Digestible energy was calculated with the formula of Rittenhouse et al. (1971): $\mathrm{DE}(\mathrm{kJ} / \mathrm{g})=[0.038 \times \operatorname{IVDMD}(\%)+0.18] \times 4.18$.

Deer were expected to have a more selective diet and choose higher quality forages than elk or cattle (Hofmann, 1989). In general, dietary CP of $7 \%$ is considered to be the minimum necessary for maintenance of a positive nitrogen balance (Murphy \& Coates, 1966) for an adult female deer. Furthermore, Amman, Cowan, Mothershead, and Baumgardt (1973) suggested that diet IVDMD should be $\geq 50 \%$, while Ullrey et al. (1970) indicated diets that contain ME concentration of $9.45 \mathrm{~kJ} / \mathrm{g}$ are considered adequate for deer. Therefore, we speculate that deer in the present study would meet their CP requirement, whereas energy may have been limited. Cook et al. (2004) categorized late-summer-early autumn non-lactating cow elk nutrition status based on diet ME as: 1) excellent $(>9.95 \mathrm{~kJ} / \mathrm{g}), 2)$ good $(9.45-9.95 \mathrm{~kJ} / \mathrm{g}), 3)$ marginal $(8.23-9.45 \mathrm{~kJ} / \mathrm{g})$, and 4) poor $(<8.23 \mathrm{~kJ} / \mathrm{g})$. Thus, elk in the current study could fall in the last category.

Our study suggested that early-summer utilization of forage by cattle or elk at the moderate level has no significant effect on the subsequent late-summer nutrient intake rate of cattle, deer, and elk. This may be attributed to several factors including study site rangeland heterogeneity (Bailey, Dumont, \& De Vries, 1998), the optimal utilization level (Johnson, 1953; Ganskopp, Svejcar, Taylor, Farstvedt, \& Paintner, 1999, animal plasticity in diet selections (Holechek \& Vavra, 1982; DelCurto et al., 2005) and intake rate (Short, 1971; Wickstrom et al., 1984; Hobbs, 1989). The prior stocking treatment was at a moderate level in this study and Skovlin et al. (1976) noted that moderate stocking maintained grazing capacity and provided acceptable cattle gains. All animal species in the current study had forage intake comparable to other studies (Cordova, Wallace, \& Pieper, 1978; Parker et al., 1999; Cook et al., 2004). Intake estimates for foraging cattle have been highly variable, but most appear to be within a range of 40 to $90 \mathrm{~g} \mathrm{DM} / \mathrm{kg}^{0.75} /$ day (Cordova et al., 1978). Others (Allden \& Whittaker, 1970; Chacon \& Stobbs, 1976) reported that maximum diet intake rate for livestock increased as a function of body weight and ranged from $4.8 \mathrm{~g} / \mathrm{min}$ in sheep to $18.0 \mathrm{~g} / \mathrm{min}$ in cattle (Allden \& 
Whittaker, 1970; Chacon \& Stobbs, 1976).

Also, Wickstrom et al. (1984) documented that forage intake rates were $0.15 \mathrm{~g} / \mathrm{kg}^{0.75} / \mathrm{min}$ for deer and 0.31 $\mathrm{g} / \mathrm{kg}^{0.75} / \mathrm{min}$ for elk. In agreement with Wickstrom et al. (1984), the current study found deer forage intake to be lower compared to elk and cattle (1.6 and 1.9 times lower, respectively). Findholt et al. (2004) in a companion study discussed how bite rate declined $(p<0.05)$ and DMI tended to decline $(p<0.20)$ on previously cattle grazed paddocks. However, our study revealed that animal nutrient intake was compensated by an elevated percentage (nutrient density) of selected diet CP and ME. Thus, these animals maintained a consistent nutrient intake rate on all grazing treatments in this study. Our results further indicated the ability of cattle and elk to change diets in response to previous grazing which may likely be the key to the animal's ability to maintain a consistent nutrient intake rate.

To understand the influences of nutrition, knowledge of standard biological measurements and baseline nutritional requirements are necessary. NRC (1996) outlined nutrient-density requirements for $453 \mathrm{~kg}$ beef cattle as $7.8 \% \mathrm{CP}$ and $8.4 \mathrm{MJ} / \mathrm{kg} \mathrm{ME}$ (daily intake $\geq 82.5 \mathrm{MJ}$ or $9.75 \mathrm{~kg} \mathrm{DM}$ ) for a $0.45 \mathrm{~kg} /$ day gain, which indicates negligible deficiency cattle $\mathrm{CP}$ in mixed-conifer rangelands in the late-summer. Due to NDF content or gastrointestinal fill (Van Soest, 1994; Mertens, 1987), cattle forage intake in our study should not exceed $9.52 \mathrm{~kg}$ DM day. Accordingly, maximum consumption would not exceed 74.0 MJ/day ME intake for cattle and this barely meets a beef cattle $0.22 \mathrm{~g} /$ day gain $(\mathrm{NRC}, 1996)$ requirement. Thus, gut fill is also likely a limiting factor for cattle diet intake during this season when using this rangeland.

The CP requirement for an adult non-lactating deer is $4.8 \mathrm{~g} / \mathrm{kg}^{0.75} /$ day (Holter, Hayes, \& Smith, 1979) and deer ME requirement is $543 \mathrm{~kJ} / \mathrm{kg}^{0.75} / \mathrm{day}$ (McCall, Brown, \& Bender, 1997). Subsequently, if we assume our observations of foraging behavior of deer were representative of foraging throughout the day, based on the consumption rate, deer needed to forage at least 10 and 12 hours, respectively, to meet their CP and ME nutritional needs. In addition, daily forage intake, as a percentage of body weight would be equal to about $2.1 \%$ and 2.5\%, if deer forage 10 and 12 hours, respectively. Others (Parker et al., 1999) have postulated that deer daily intake of $2.5 \%$ of body weight or higher would be expected during summer when forage quality is normally high. Krysl and Hess (1993) in their study on foraging behavior, indicated that daily foraging time for cattle ranges from 6 to 13 hours/day. Likewise, Parker et al. (1999) found that time spent grazing by black-tailed deer on Channel Island averaged 11.5 hours. In contrast, on summer range in the Sierra Nevada of California, female deer foraged 7.7 hours (Kie, Evans, Loft, \& Menke, 1991). Nevertheless, as Canon, Urness, and DeBule (1987) indicated, the upper limit of foraging time is about 13 hours in ruminants.

Elk requirements for protein and energy have been studied extensively (Cook et al., 2004). In late-summer, an adult non-lactating female elk requires $836 \mathrm{~kJ} / \mathrm{kg}^{0.75} /$ day ME for maintenance (normal metabolic rate plus activity; Cook, 2002). Also, females need an additional $92 \mathrm{~kJ} / \mathrm{kg}^{0.75} /$ day or $230 \cdot \mathrm{kJ} / \mathrm{kg}^{0.75} /$ day ME for replenishing $10 \%$ (mild winter) or $25 \%$ (harsh winter) body weight, respectively, for winter-catabolized tissue loss (Jiang \& Hudson, 1992; Cook, 2002). The daily minimum CP requirement for elk $7.0 \mathrm{~g} / \mathrm{kg}^{0.75} / \mathrm{day}$ CP for live-weight maintenance, and 0.72 and $1.81 \mathrm{~g} / \mathrm{kg}^{0.75} /$ day $\mathrm{CP}$, respectively, for replacement of $10 \%$ and $25 \%$ winter catabolized body weight loss. Therefore, cow elk foraging with the same nutrient intake rate as in the current study, would need a minimum of 12-13 and 13-15 hours foraging time to cover daily CP and ME requirements, respectively. Thus, our study suggests that elk in mixed-conifer rangelands may also be unable to meet their energy requirements in late-summer regardless of previous grazing.

\section{Conclusions and Recommendations}

Early-summer grazing by cattle or elk at the moderate utilization level did not affect the subsequent nutrient intake rate of cattle, deer, and elk. In late-summer, cattle and elk were able to maintain dietary CP and ME by increasing their shrub and forb consumptions in response to previous grazing by cattle. Furthermore, the study demonstrated that in late-summer on mixed-conifer rangelands energy density is a limiting factor for all three of the ungulates. Therefore, if the management goal is high productivity of ungulates, it may be necessary to implement alternative range improvement practices like range rotation of cattle, overstory reduction or fire management strategies that enhance forage quality and quantity. Overstory reduction treatments would have to result in an increase in palatable shrubs in order to improve late season forage quality. However, more research is needed regarding the long term effects of summer cattle grazing and use by wild ungulates of rangeland resources at different utilization level, at larger scales.

\section{Acknowledgments}

This research was funded by the Eastern Oregon Agricultural Research Center, Rocky Mountain Elk Foundation, and the U.S. Department of Agriculture, Forest Service, Pacific Northwest Research Station. Implementation of 
this research was completed under provisions of the Federal Aid in Wildlife Restoration Act (Pittman-Robertson Act), administered by the Oregon Department of Fish and Wildlife. This manuscript greatly benefited from reviews by David Bohnert (Eastern Oregon Agricultural Research Center, Oregon, USA) and Enkhjargal Darambazar (University of Saskatchewan, Saskatoon, SK, Canada). We are indebted to P. Coe, J. Noyes, J. Paustian, and R. Stussy of the Oregon Department of Fish and Wildlife for assistance in the field.

\section{References}

Allden, W. G., \& Whittaker, I. A. M. (1970). The determinants of herbage intake by grazing sheep: the interrelationship of factors influencing herbage intake and availability. Australian Journal of Agricultural Research, 21, 755-766. https://doi.org/10.1071/AR9700755

Amman, A. P., Cowan, R. L., Mothershead, C. L., \& Baumgardt, B. R. (1973). Dry matter and energy intake in relation to digestibility in white-tailed deer. Journal of Wildlife Management, 37, 195-201. https://doi.org/10.2307/3798904

Anderson, E. W., \& Scherzinger. R. J. (1975). Improving quality of winter forage for elk by cattle grazing. Journal of Range Management, 28, 120-125. https://doi.org/10.2307/3897442

AOAC. (1990). Official methods of analysis. 15th edn. Arlington, VA: Association of Official Analytical Chemists. pp. 1175.

Bailey, D. W., Dumont, B., \& De Vries, M. F. (1998). Utilization of heterogeneous grasslands by domestic herbivores: Theory to management. Annales de Zootechnie, 47, 321-333. https://doi.org/10.1051/animres:19980501

Canon, S. K., P. J. Urness, P. J., \& DeBule, N. V. (1987). Foraging behavior and dietary nutrition of elk in burned aspen forest. Journal of Range Management, 40, 433-438. https://doi.org/10.2307/3899605

Chacon, E., \& Stobbs. T. H. (1976). Influence of progressive defoliation of a grass sward on the eating behaviour of cattle. Australian Journal of Agricultural Research, 27, 709-727. https://doi.org/10.1071/AR9760709

Clark, P. E., Krueger, W. C., Bryant, L. D., \& Thomas, D. R. (2000). Livestock grazing effects on forage quality of elk winter range. Journal of Range Management, 53, 97-105. https://doi.org/10.2307/4003399

Coe, P. E., Johnson, B. K., Kern, J. W., Findholt, S. L., Kie, J. G., \& Wisdom, M. J. (2001). Responses of elk and mule deer to cattle in summer. Journal of Range Management, 54, A51-76.

Coe, P. K., Johnson, B. K., Stewart, K. M., \& Kie, J. G. (2004). Spatial and temporal interactions of elk, mule deer, and cattle. Transactions of the North American Wildlife and Natural Resources Conference, 69, 656-669.

Cook, C. W., \& Stubbendieck, J. (Eds.) (1986). Range research: basic problems and techniques. pp. 317. (Society for Range Management: Denver).

Cook, J. G. (2002). Nutrition and food habits. In D. E. Toweill \& J. W. Thomas (Eds.), North American elk: ecology and management (pp. 259-351). Smithsonian Institution Press: Washington and London.

Cook, J. G., Johnson, B. K., Cook, R. C., Riggs, R. A., DelCurto, T., Bryant, L. D., \& Irwin, L. L. (2004). Effects of summer-autumn nutrition and parturition date on reproduction and survival of elk. Wildlife Monographs, 155,61 .

Cordova, F. J., Wallace, J. D., \& Pieper, R. D. (1978). Forage intake by grazing livestock: A review. Journal of Range Management, 31, 430-478. https://doi.org/10.2307/3897201

Crane, K. K., Mosley, J. C., Brewer, T. K., Torstenson, W. L. F., \& Tess, M. W. (2001). Influence of cattle grazing on elk habitat selection. In: 'Proceedings of Western Section American Society of Animal Sciences' pp. 160-164.

Damiran, D. (2006). Influence of previous cattle and elk grazing on the subsequent diet quality and nutrient intake rate of cattle, deer, and elk grazing late-summer mixed-confier rangelands (Unpublished doctoral dissertation). Oregon State University, Corvallis, USA.

Damiran, D., DelCurto, T., Bohnert, D. W., \& Findholt, S. L. (2008). Comparison of techniques and grinding sizes to estimate digestibility of forage based ruminant diets. Animal Feed Science and Technology, 141, 15-35. https://doi.org/10.1016/j.anifeedsci.2007.04.007

Damiran, D., DelCurto, T., Findholt, S. L., Johnson, B., \& Vavra, M. (2012). Comparison of bite-count and rumen evacuation techniques to estimate diet quantity and quality in grazing cattle. Rangeland Ecology and 
Management, 66, 106-109. https://doi.org/10.2111/REM-D-12-00046.1

Darambazar, E., DelCurto, T., \& Damiran, D. (2013). Changes in forage quantity and quality with continued late-summer cattle grazing a riparian pasture in Eastern Oregon of United States. Sustainable Agriculture Research, 2(4), 64-76. https://doi.org/10.5539/sar.v2n4p64

DelCurto, T., Porath, M., Parsons, C. T., \& Morrison, J. (2005). Management strategies for sustainable beef cattle grazing on forested rangelands in the Pacific Northwest. Rangeland Ecology and Management, 58, 119-127. https://doi.org/10.2111/1551-5028(2005)58<119:MSFSBC >2.0.CO;2

Findholt, S. L., Johnson, B. K., Damiran, D., DelCurto, T., \& Kie, J. G. (2004). Diet composition, dry matter intake, and diet overlap of mule deer, elk, and cattle. Transactions of the North American Wildlife and Natural Resources Conference, 69, 670-686.

Ganskopp, D., Svejcar, T., Taylor, F., Farstvedt, J., \& Paintner, K. (1999). Seasonal cattle management in 3- to 5-year-old bitterbrush stands. Journal of Range Management, 52, 166-173. https://doi.org/10.2307/4003512

Hobbs, N. T. (1989). Linking energy balance to survival in mule deer: development and test of a simulation model. Wildlife Monographs, 101, 39.

Hofmann, R. R. (1989). Evolutionary steps of ecophysiological adaptation and diversification of ruminants: a comparative view of their digestive system. Anatomy of the gastrointestinal tract. Oecologia, 78, 443-457. https://doi.org/10.1007/BF00378733

Holechek, J. L. \& Vavra, M. (1983). Drought effects on diet and weight gains of yearling heifers in Northeastern Oregon. Journal of Range Management, 36, 227-231. https://doi.org/10.2307/3898169

Holechek, J. L., \& Vavra, M. (1982). Forage intake by cattle on forest and grassland range. Journal of Range Management, 35, 737-741. https://doi.org/10.2307/3898252

Holter, J. B., Hayes, H. H., \& Smith, S. H. (1979). Protein requirement of yearling white-tailed deer. Journal of Wildlife Management, 43, 872-879. https://doi.org/10.2307/3808270

Jiang, A., \& Hudson R. J. (1992). Estimating forage intake and energy requirements of free-ranging wapiti (Cervus elaphus). Canadian Journal of Zoology, 70, 675-679. https://doi.org/10.1139/z92-101

Johnson, W. M. (1953). Effects of grazing intensity upon vegetation and cattle gains on ponderosa pine-bunchgrass of the front range of Colorado. Circular 929. Washington, DC: U. S. Department of Agriculture, Forest Service. pp. 36.

Kie, J. G., Evans, C. J., Loft, E. R., \& Menke, J. W. (1991). Foraging behavior by mule deer: The influence of cattle crazing. Journal of Wildlife Management, 55, 665-674. https://doi.org/10.2307/3809516

Krysl, L. J., \& Hess, B. W. (1993). Influence of supplementation on behavior of grazing cattle. Journal of Animal Science, 71, 2546-2555. https://doi.org/10.2527/1993.7192546x

Marten, G. C., \& Barnes, R. F. (1980). Prediction of energy digestibility of forages with in vitro rumen fermentation and fungal enzyme systems. In: 'Standardization of analytical methodology for feeds'. (Eds.W. G. Pigden, C. C. Balch, and M. Graham) Ottawa, ON: International Research Center, pp. 61-71.

McCall, T. C., Brown, R. D., \& Bender, L. C. (1997). Comparison of technique for determining the nutritional carrying capacity for white-tailed deer. Journal of Range Management, 50, 33-38. https://doi.org/10.2307/4002702

Mertens, D. R. (1987). Predicting intake and digestibility using mathematical models of ruminal function. Journal of Animal Science, 64, 1548-1558. https://doi.org/10.2527/jas1987.6451548x

Murphy, D. A., \& Coates, J. A. (1966). Effects of dietary protein on deer. Transactions of the North American Wildlife and Natural Resources Conference, 31, 129-139.

NADP (National Atmospheric Deposition Program) (2012). Automated weather data network, Champaign IL, United States. Retrieved from http://nadp.slh.wisc.edu/ nadp data, Accessed 15 July 2019

NRC (National Research Council) (1996). Nutrient requirements of beef cattle. Update 2000. 7th edn. (National Academy Press: Washington, DC).

Parker, K. L., Gillingham, M. P., Hanley, T. A., \& Robbins, C. T. (1999). Energy and protein balance of free-ranging black-tailed deer in a natural forest environment. Wildlife Monographs, 143, 48.

Peek, J. M., \& Krausman, P. R. (1996). Grazing and mule deer. In P. R. Krausman (Eds.), Rangeland wildlife (pp. 
183-192). Society Rangeland Management: Denver.

Rittenhouse, L. R. (2000). Supplementation in an uncertain environment: dynamics of the grazing resources. In (Ed.), Strategic supplementation of beef cattle consuming low-quality roughages in the western United States (pp. 117-123). Oregon State University, Agricultural Experimental Station: Corvallis, OR.

Rittenhouse, L. R., Streeter, C. L., \& Clanton, D. C. (1971). Estimating digestible energy from digestible dry and organic matter in diets of grazing cattle. Journal of Range Management, 24, 73-75.

https://doi.org/10.2307/3896072

SAS. (2002). SAS/STAT guide for personal computer. Version 9.3. (SAS Institute: Cary, NC.).

Short, H. L. (1971). Forage digestibility and diet of deer on southern upland range. Journal of Wildlife Management, 35, 698-706. https://doi.org/10.2307/3799775

Skovlin, J. M., Harris, R. W., Strickler, G. S., \& Garrison, G. A. (1976). Effect of cattle grazing methods on ponderosa pine-bunchgrass range in the Pacific Northwest. Technical Bulletin No. 1531. pp.39. (U. S. Department of Agriculture, Forest Service, Pacific Northwest Forest and Range Experiment Station: La Grande, OR.).

Stewart, K. M., Bowyer, R. T., Kie, J. G., Cimon, N., \& Johnson, B. K. (2002). Temporospatial distributions of elk, mule deer, and cattle: resource partitioning and competitive displacement. Journal of Mammalogy, 83, 229-244. https://doi.org/10.1093/jmammal/83.1.229

Svejcar, T., \& Vavra, M. (1985). The influence of several range improvements on estimated carrying capacity and potential beef production. Journal of Range Management, 38, 395-399. https://doi.org/10.2307/3899706

Ullrey, D. E., Youatt, W. G., Johnson, H. E., Fay, L. D., Schoepke, B. L., \& Magee, W. T. (1970). Digestible and metabolizable energy requirements for winter maintenance of Michigan white-tailed does. Journal of Wildlife Management, 34, 863-869. https://doi.org/10.2307/3799155

USDA NRCS (United States Department of Agriculture, Natural Resources Conservation Service. (2012). The PLANTS database, Version 3.5. Retrieved from http://plants.usda.gov

Van Soest, P. J. (1994). Nutritional ecology of the ruminant. 2nd edn. (Cornell University Press: Ithaca, NY.)

Van Soest, P. J., Robertson, J. B., \& Lewis, B. A. (1991). Methods for dietary fiber, neutral detergent fiber, and nonstarch polysaccharides in relation to animal nutrition. Journal of Dairy Science, 74, 3583-3597. https://doi.org/10.3168/jds.S0022-0302(91)78551-2

Wallis De Vries, M. F. (1995). Estimating forage intake and quality in grazing cattle: a reconsideration of the hand-plucking method. Journal of Range Management, 48, 370-375. https://doi.org/10.2307/4002491

Wickstrom, M. L., Robbins, C. T., Hanley, T. A., Spalinger, D. E., \& Parish, S. M. (1984). Food intakes and foraging energetics of elk and mule deer. Journal of Wildlife Management, 48, 1285-1301. https://doi.org/10.2307/3801789

Wisdom, M. J., \& Thomas, J. W. (1996). Elk. In P. R. Krausman (Eds.), Rangeland wildlife (pp. 157-182). Society Rangeland Management: Denver.

\section{Copyrights}

Copyright for this article is retained by the author(s), with first publication rights granted to the journal.

This is an open-access article distributed under the terms and conditions of the Creative Commons Attribution license (http://creativecommons.org/licenses/by/3.0/). 